\title{
The L10P Polymorphism and Serum Levels of Transforming Growth Factor $\beta 1$ in Human Breast Cancer
}

\author{
Eva Taubenschuß ${ }^{1}$, Erika Marton ${ }^{1}$, Maurice Mogg ${ }^{1}$, Barbara Frech ${ }^{1}$, Lisa Ehart ${ }^{1}$, \\ Dana Muin ${ }^{1}$ and Martin Schreiber ${ }^{1,2, *}$
}

1 Department of Obstetrics and Gynecology, Medical University of Vienna, Waehringer Guertel 18-20, A-1090 Vienna, Austria; E-Mails: eva.taubenschuss@gmx.at (E.T.); erika.marton@meduniwien.ac.at (E.M.); maurice.mogg@gmail.com (M.M.); barbara.frech@gmx.net (B.F.); lisa.ehart@gmx.at (L.E.); dana.muin@meduniwien.ac.at (D.M.)

2 Comprehensive Cancer Center (CCC), Medical University of Vienna, A-1090 Vienna, Austria

* Author to whom correspondence should be addressed; E-Mail: martin.schreiber@meduniwien.ac.at; Tel.: +43-1-40400-2738; Fax: +43-1-40400-7842.

Received: 21 June 2013; in revised form: 13 July 2013 / Accepted: 15 July 2013 /

Published: 24 July 2013

\begin{abstract}
The L10P single nucleotide polymorphism (SNP) is located in the signal sequence of the transforming growth factor $\beta 1$ (TGF $\beta 1$ ) gene. The proline-encoding (Pro-) allele of this SNP has been associated with an increased breast cancer risk, which has been attributed to the elevated secretion of this TGF $\beta 1$ variant observed in vitro and in male subjects. Here we investigated the association of the L10P SNP with serum levels of TGF $\beta 1$ in female breast cancer patients and controls. We genotyped the L10P SNP in 276 breast cancer patients and 255 controls. Serum TGF $\beta 1$ concentrations were measured by enzyme-linked immunosorbent assay (ELISA) in a subset of the study population $(n=211)$. We found no evidence for an association of the L10P SNP with breast cancer risk (per-allele odds ratio: 0.91; 95\% confidence interval: 0.71-1.16). However, patients with the Pro/Pro genotype exhibited a significantly younger age at breast cancer onset $(55.2 \pm 14.3$ years $)$ than Leu/Leu patients $(60.6 \pm 13.6$ years; $p=0.04)$, which may reflect the ability of TGF $\beta$ to promote tumor progression. Mean TGF $\beta 1$ serum levels of Pro-allele carriers were $39.4 \pm 7.4 \mathrm{ng} / \mathrm{mL}$, whereas those of Leu/Leu subjects were $37.6 \pm 6.0 \mathrm{ng} / \mathrm{mL}$ $(p=0.07)$. Thus, compared to a previous study of male subjects, we observed only a modest increase, if any, in TGF $\beta 1$ levels of female Pro-allele carriers.
\end{abstract}

Keywords: breast cancer; TGF $\beta 1$; rs1800470; L10P SNP; serum levels 


\section{Introduction}

Transforming growth factor $\beta$ (TGF $\beta$ ) is a multifunctional cytokine and a key growth suppressor in many cell types, eliciting potent anti-proliferative and apoptotic responses [1-3]. Accordingly, loss-of-function mutations in the TGF $\beta$ pathway have been frequently observed in human tumors, classifying it as a tumor suppressor. However, at later stages of cancer progression tumor cells often develop resistance to the tumor suppressive activity of TGF $\beta$. In tumor cells that are thus relieved from its growth inhibitory function, TGF $\beta$ can actively promote tumor progression, particularly by enhancing the invasiveness and metastatic propensity $[2,4,5]$. Whereas this dual role of TGF $\beta$ as a tumor suppressor and pro-tumorigenic factor is well understood in various model systems, it is less clear if and at which stage this switch from anti- to pro-tumorigenic activity of TGF $\beta$ occurs in human breast cancer.

The gene encoding TGF $\beta 1$, one of the three human TGF $\beta$ isoforms, contains a single-nucleotide polymorphism (SNP) at position 29 of its coding sequence, the major allele encoding leucine and the minor allele encoding proline as amino acid 10 (Leu10Pro; $\mathrm{T}+29 \mathrm{C}$; rs1800470; hereafter referred to as L10P). A large study of the Breast Cancer Association Consortium (BCAC) has reported an association of the Pro-allele with a moderate, but significantly increased, breast cancer risk (e.g., Pro/Pro vs. Leu/Leu: OR, 1.16; 95\% confidence interval, 1.08-1.25 [6]). Other studies have either reported an increased risk, an unaltered risk, or even a decreased risk associated with the Pro-allele [7-24]. Shin et al. [19] suggested that these inconsistent results are due to the dual role of TGF $\beta$, and that the Pro-allele may reduce the incidence of early-stage breast cancer, but promote the progression of late-stage breast cancer. In their study the Pro-allele was associated with a decreased risk of early-stage ( 0 or I), but a (non-significantly) increased risk of advanced-stage (III or IV) breast cancer [19]. The association of other TGF $\beta 1$ SNPs with breast cancer has also been analysed, such as the promoter SNPs C-509T and G-800A and the R25P coding SNP [7-13].

The L10P SNP is located in the signal sequence of $T G F \beta 1$, and has been suggested to affect the efficiency of TGF $\beta 1$ secretion [7]. Transfections of HeLa cells with expression vectors encoding the Pro-allele resulted in a more than two-fold higher secretion of TGF $\beta 1$ into the culture medium than parallel transfections with the Leu-allele [7]. Accordingly, the Pro-allele is considered a "high-activity" (hypermorphic) allele compared to the Leu-allele. The in vivo association of the L10P genotype with TGF $\beta 1$ serum levels has been investigated in a Japanese study of human myocardial infarction [25]. In this study, the serum concentrations of TGF $\beta 1$ were significantly higher in male patients and controls with the Pro/Pro vs. the Leu/Leu genotype. However, correlations of TGF $\beta 1$ serum levels with L10P genotypes in female patients or controls have not been not reported so far [25].

\section{Results and Discussion}

\subsection{The TGF $\beta 1$ LIOP SNP and Breast Cancer Risk}

The L10P SNP of TGF $\beta 1$ was genotyped in 274 breast cancer patients and 252 female controls. The frequencies of the L10P genotypes in patients and controls, and clinical characteristics of the patients are shown in Table 1. The frequency of the Pro-allele was $40.0 \%$ in patients and $42.3 \%$ in controls. 
Table 1. Clinical characteristics of the study population and frequency of the TGF $\beta 1$ L10P genotypes in the indicated subpopulations.

\begin{tabular}{|c|c|c|c|c|c|c|}
\hline & & Total & Leu/Leu & Leu/Pro & Pro/Pro & $p$-value \\
\hline All subjects & & 526 & $186(35.4 \%)$ & $248(47.1 \%)$ & $92(17.5 \%)$ & \\
\hline Patients & & 274 & $99(36.1 \%)$ & $131(47.8 \%)$ & $44(16.1 \%)$ & 0.664 \\
\hline Controls & & 252 & $87(34.5 \%)$ & $117(46.4 \%)$ & $48(19.0 \%)$ & \\
\hline \multicolumn{7}{|l|}{ Patient subgroups } \\
\hline \multirow[t]{4}{*}{ Tumor size } & pT1 & 133 & $50(37.6 \%)$ & $62(46.6 \%)$ & $21(15.8 \%)$ & 0.586 \\
\hline & pT2 & 55 & $20(36.4 \%)$ & $27(49.1 \%)$ & $8(14.5 \%)$ & \\
\hline & pT3, pT4 & 12 & $3(25.0 \%)$ & $5(41.7 \%)$ & $4(33.3 \%)$ & \\
\hline & other, na & 74 & $26(35.1 \%)$ & $37(50.0 \%)$ & $11(14.9 \%)$ & \\
\hline \multirow[t]{3}{*}{ Tumor type } & ductal & 148 & $53(35.8 \%)$ & $71(48.0 \%)$ & $24(16.2 \%)$ & 0.561 \\
\hline & lobular & 48 & $20(41.7 \%)$ & $23(47.9 \%)$ & $5(10.4 \%)$ & \\
\hline & other, na & 78 & $26(33.3 \%)$ & $37(47.4 \%)$ & $15(19.2 \%)$ & \\
\hline \multirow[t]{4}{*}{ Stage } & $\mathrm{I}$ & 97 & $38(39.2 \%)$ & $40(41.2 \%)$ & $19(19.6 \%)$ & 0.290 \\
\hline & II & 63 & $22(34.9 \%)$ & $35(55.6 \%)$ & $6(9.5 \%)$ & \\
\hline & III & 18 & $5(27.8 \%)$ & $10(55.6 \%)$ & $3(16.7 \%)$ & \\
\hline & other, na & 96 & $34(35.4 \%)$ & $46(47.9 \%)$ & $16(16.7 \%)$ & \\
\hline \multirow[t]{4}{*}{ Grade } & pG1 & 42 & $17(40.5 \%)$ & $21(50.0 \%)$ & $4(9.5 \%)$ & 0.405 \\
\hline & pG2 & 115 & $38(33.0 \%)$ & $58(50.4 \%)$ & $19(16.5 \%)$ & \\
\hline & pG3 & 88 & $36(40.9 \%)$ & $35(39.8 \%)$ & $17(19.3 \%)$ & \\
\hline & na & 29 & $8(27.6 \%)$ & $17(58.6 \%)$ & $4(13.8 \%)$ & \\
\hline \multirow[t]{3}{*}{ Lymph node status } & $\mathrm{pN} 0$ & 143 & $56(39.3 \%)$ & $62(43.4 \%)$ & $25(17.5 \%)$ & 0.320 \\
\hline & $\mathrm{pN}+$ & 53 & $18(34.0 \%)$ & $29(54.7 \%)$ & $6(11.3 \%)$ & \\
\hline & na & 78 & $25(32.1 \%)$ & $40(51.3 \%)$ & $13(16.7 \%)$ & \\
\hline \multirow[t]{3}{*}{ ER status } & pos & 201 & $75(37.3 \%)$ & $100(49.8 \%)$ & $26(12.9 \%)$ & 0.085 \\
\hline & neg & 61 & $21(34.4 \%)$ & $25(41.0 \%)$ & $15(24.6 \%)$ & \\
\hline & na & 12 & $3(25.0 \%)$ & $6(50.0 \%)$ & $3(25.0 \%)$ & \\
\hline \multirow[t]{3}{*}{ PR status } & pos & 138 & $47(34.1 \%)$ & $73(52.9 \%)$ & $18(13.0 \%)$ & 0.130 \\
\hline & neg & 117 & $46(39.3 \%)$ & $48(41.0 \%)$ & $23(19.7 \%)$ & \\
\hline & na & 19 & $6(31.6 \%)$ & $10(52.6 \%)$ & $3(15.8 \%)$ & \\
\hline \multirow[t]{3}{*}{ HER2 status } & pos & 51 & $24(47.1 \%)$ & $21(41.2 \%)$ & $6(11.8 \%)$ & 0.176 \\
\hline & neg & 201 & $67(33.3 \%)$ & $99(49.3 \%)$ & $35(17.4 \%)$ & \\
\hline & na & 22 & $8(36.4 \%)$ & $11(50.0 \%)$ & $3(13.6 \%)$ & \\
\hline \multirow[t]{3}{*}{ p53 status } & pos & 57 & $23(40.4 \%)$ & $22(38.6 \%)$ & $12(21.1 \%)$ & 0.296 \\
\hline & neg & 190 & $68(35.8 \%)$ & $94(49.5 \%)$ & $28(14.7 \%)$ & \\
\hline & na & 27 & $8(29.6 \%)$ & $15(55.6 \%)$ & $4(14.8 \%)$ & \\
\hline
\end{tabular}

Numbers of patients in each of the indicated subgroups are shown. Numbers in parentheses indicate the fraction of patients in each row with genotypes Leu/Leu, Leu/Pro and Pro/Pro, respectively. na: status not available; ER: estrogen receptor; PR: progesterone receptor. $p$-Values were calculated with chi-square tests of the specified subgroups (excluding na subjects). 
The controls and the breast cancer patients were both in Hardy-Weinberg equilibrium $(p=0.50$ and $p=0.96$ respectively). The Pro/Pro genotype was slightly less frequent in breast cancer patients than in controls (16.1\% and 19.0\%, respectively; Table 1). On the other hand, the fraction of patients with the Pro/Pro genotype tended to be increased in several patient subgroups associated with advanced cancer progression and/or poor prognosis. For example, $19.3 \%$ of grade $3 v s .14 .6 \%$ of grade $1 / 2 ; 24.6 \%$ of estrogen receptor (ER) negative vs. $12.9 \%$ of ER positive; and $19.7 \%$ of progesterone receptor (PR) negative $v$ s. $13.0 \%$ of PR positive patients exhibited the Pro/Pro genotype (Table 1). However, none of these differences in genotype distribution among clinical subgroups were statistically significant (Table 1).

Next, odds ratios and 95\% confidence intervals for breast cancer risk were determined. All comparisons revealed odds ratios close to unity, and any deviations from unity were not significant (Table 2). Thus, the Pro/Pro genotype, like the Pro-allele, was not associated with an increased breast cancer risk. We next analyzed the association of the L10P genotype with the age at breast cancer diagnosis. Interestingly, the mean age at breast cancer onset of patients with the Pro/Pro genotype was $55.2 \pm 14.3$ years (median age, 57.0 years), whereas that of Leu/Leu patients was $60.6 \pm 13.6$ years (median age, 62.4 years; $p=0.04$; Figure 1A). Leu/Pro patients were diagnosed at an intermediate mean age ( $58.5 \pm 14.1$ years; median age, 59.3 years; Figure $1 \mathrm{~A})$. Comparison of the cumulative breast cancer incidence of all three genotypes by a log rank test also revealed significant differences $(p=0.048$; Figure 1B).

Table 2. Odds ratios and $95 \%$ confidence intervals for $T G F \beta 1 \mathrm{~L} 10 \mathrm{P}$ genotypes or alleles and breast cancer risk.

\begin{tabular}{cccc}
\hline Genotypes/Alleles & OR & $\mathbf{9 5 \%}$ c.i. & $\boldsymbol{p}$-Value \\
\hline Pro/Pro $v s$. Leu/Leu & 0.81 & $0.49-1.33$ & 0.409 \\
Pro/Pro $v s$. Leu/Pro & 0.82 & $0.51-1.32$ & 0.429 \\
Leu/Pro $v s$. Leu/Leu & 0.98 & $0.67-1.44$ & 0.934 \\
Pro/Pro + Leu/Pro $v s$. Leu/Leu & 0.93 & $0.65-1.33$ & 0.700 \\
Pro/Pro $v s$. Leu/Pro + Leu/Leu & 0.81 & $0.52-1.28$ & 0.368 \\
Pro $v s$. Leu & 0.91 & $0.71-1.16$ & 0.455 \\
\hline
\end{tabular}

Analyses of breast cancer cases vs. controls of the indicated genotypes or Pro vs. Leu alleles are shown. c.i.: confidence interval.

\subsection{Analysis of TGFß1 Serum Levels}

Next, serum levels of TGF $\beta 1$ protein were analyzed in breast cancer patients $(n=110)$ and control subjects $(n=101)$ with known L10P genotypes (Figure 2$)$. We measured TGF $\beta 1$ serum concentrations of $37.9 \pm 7.1 \mathrm{ng} / \mathrm{mL}$ in the patients, which was significantly different from the levels measured in the controls $(39.8 \pm 6.8 \mathrm{ng} / \mathrm{mL} ; p=0.048)$. Patients with the Leu/Leu genotype $(n=36)$ had mean TGF $\beta 1$ serum levels of $36.5 \pm 6.0 \mathrm{ng} / \mathrm{mL}$, whereas mean serum levels of Pro-carriers (Leu/Pro or Pro/Pro; $n=74$ ) were $38.6 \pm 7.4$ ( $p=0.12$; Figure 2$)$. In the control population, mean TGF $\beta 1$ levels of Leu/Leu individuals $(n=33)$ were $38.8 \pm 5.8 \mathrm{ng} / \mathrm{mL}$, and of Pro-carriers $(n=68) 40.3 \pm 7.3 \mathrm{ng} / \mathrm{mL}(p=0.30)$. Thus, Pro carriers had slightly higher mean TGF $\beta 1$ serum concentrations than Leu/Leu individuals both in the control group and in the breast cancer patients, although the observed differences were not 
significant (Figure 2). When patients and control subjects were combined, mean TGF $\beta 1$ levels of Leu/Leu individuals $(n=69)$ were determined to be $37.6 \pm 6.0 \mathrm{ng} / \mathrm{mL}$, and those of Pro carriers $(n=142)$ were $39.4 \pm 7.4 \mathrm{ng} / \mathrm{mL}(p=0.07)$. Furthermore, no significant differences in TGF $\beta 1$ serum levels were observed between patients with ER negative vs. ER positive, ductal vs. lobular, pN0 vs. $\mathrm{pN}+$, pT1 vs. pT2-4 tumors, or between patients younger or older than 55 years (data not shown).

Figure 1. Breast cancer patients with the $T G F \beta 1$ Pro/Pro genotype exhibit a younger age at onset. (a) Boxplot of the age at diagnosis of patients with genotypes Leu/Leu $(n=99)$, Leu/Pro $(n=131)$, and Pro/Pro $(n=44)$; (b) Curves of the cumulative breast cancer incidence at the indicated ages at onset of patients with genotypes Leu/Leu, Leu/Pro, and Pro/Pro.
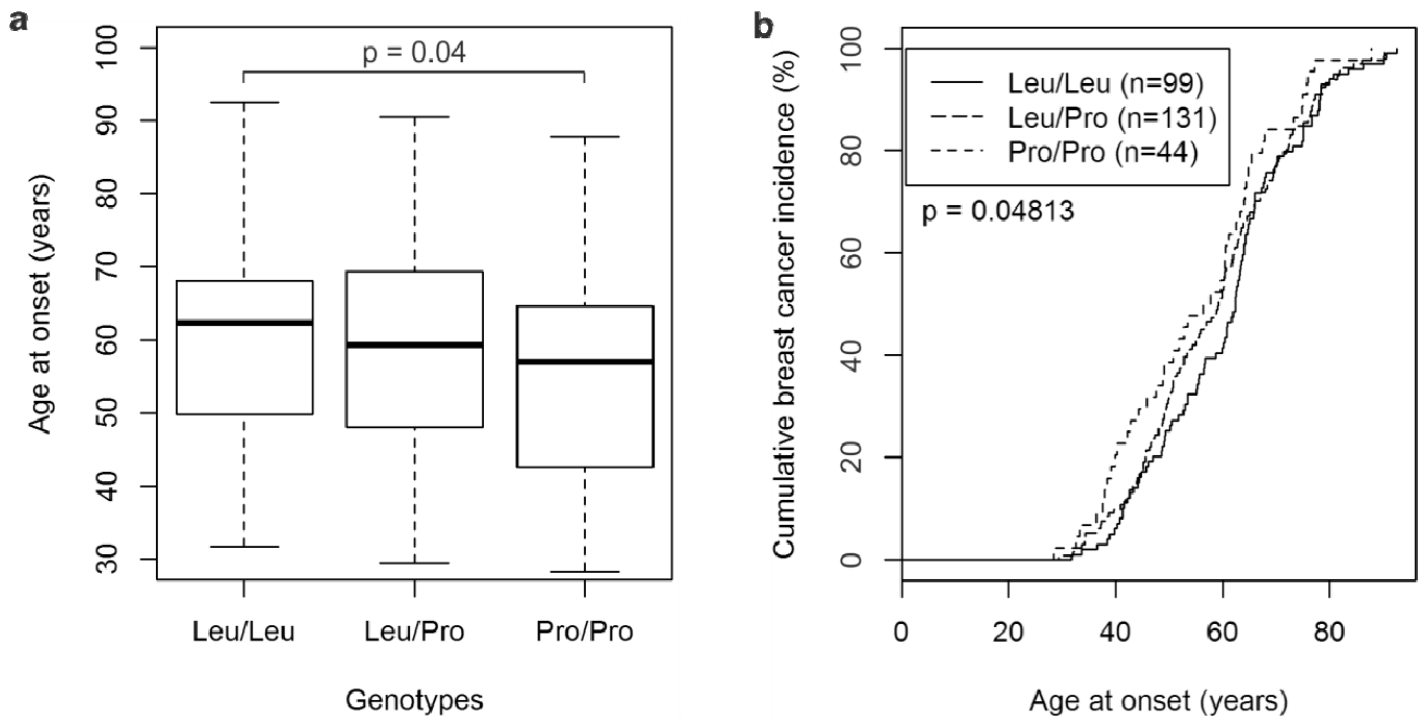

\subsection{Discussion}

The L10P SNP of TGF 1 has been extensively studied, and is characterized by contradictory reported results: some studies showed an increased breast cancer risk associated with the Pro-allele [6-9], others a decreased risk [10], and yet others-including this study (Table 2) — have found no evidence of a significantly altered risk [11-20]. These inconsistent results have been attributed to the unique dual role of TGF $\beta$, which is thought to act as a tumor suppressor during early cancer stages, but to promote cancer progression at late stages $[1,2,4,19]$. Whereas there is extensive evidence for this dual role of TGF $\beta$ in model systems, it is less clear at which stage this switch from tumor suppressor to progression factor occurs in human breast cancer and in which tumor subclass(es) it has already occurred at the time of diagnosis [2,3,19]. It has been suggested that the Pro-allele is associated with a reduced risk of in situ tumors, but an increased risk of invasive breast cancer; or with a reduced risk of early-stage invasive breast cancer, but an increased risk of breast cancer with advanced stages [6,19].

The L10P Pro-allele has been suggested to be a hypermorphic (high-activity) allele of TGF $\beta 1$. A proline residue at position 10, which is located in the signal sequence of $T G F \beta 1$, is thought to increase the efficiency of TGF $\beta 1$ secretion [7]. Expression of the Pro-allele resulted in a more than two-fold higher secretion of TGF $\beta 1$ than expression of the Leu-allele in in vitro transfection experiments [7]. 
The in vivo serum concentrations of TGF $\beta 1$ were significantly higher in male myocardial infarction patients and controls with the Pro/Pro genotype than in subjects with the Leu/Leu genotype (>50 vs. $<40 \mathrm{ng} / \mathrm{mL}$ ), whereas TGF $\beta 1$ serum levels in female patients or controls were not reported [25]. Interestingly, in this study the Pro-allele was significantly more frequent in male, but not in female patients compared to controls, suggesting a differential effect of the L10P genotype in male vs. female subjects [25]. In a gastric cancer study, TGF $\beta 1$ serum levels did not correlate with L10P genotypes [26]. To the best of our knowledge, our study is the first to correlate L10P genotypes with TGF $\beta 1$ serum levels in breast cancer patients and exclusively female controls. We observed moderately elevated serum levels in patients and controls with one or two Pro-alleles, although this trend was not significant at the $p<0.05$ level (Figure 2). Collectively, the findings by us and others indicate that the Pro-allele may also lead to higher TGF $\beta 1$ secretion in vivo, but that the observed effects on serum levels are less pronounced and more heterogeneous than in vitro.

Figure 2. Boxplot of TGFß1 serum concentrations in patients and control subjects with the Leu/Leu genotype, and the Leu/Pro or Pro/Pro genotype (Pro-carrier).

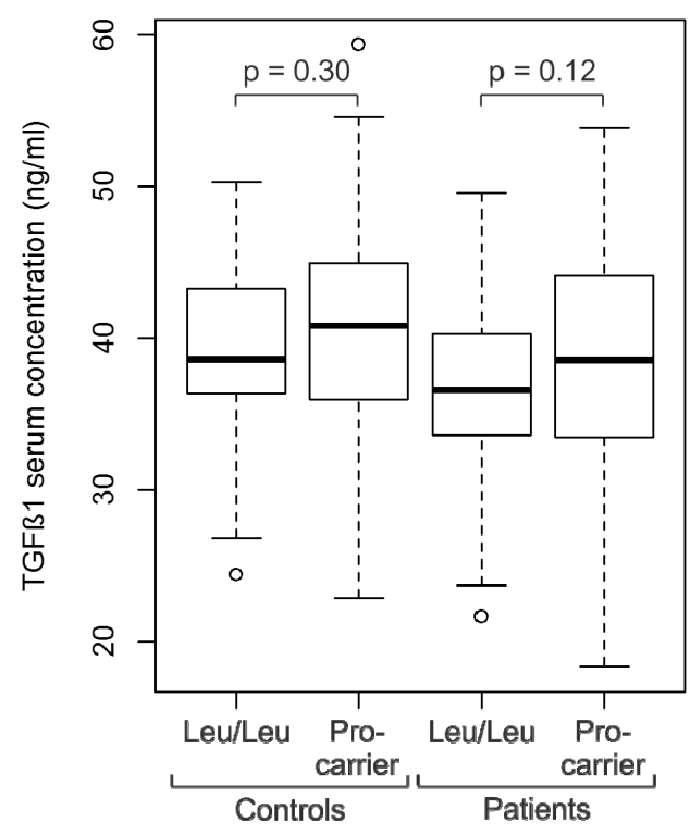

We hypothesize that, if the Pro-allele is indeed hypermorphic it should be associated with a reduced overall breast cancer incidence, due to the tumor suppressor activity of TGF $\beta$ initially effective in all tumors during the early phases of the multi-step progression. This is reflected by odds ratios below unity reported by some studies, and a particularly pronounced reduction in breast cancer risk in a prospective study (Pro/Pro vs. Leu-carriers: HR, 0.36; 95\% confidence interval (c.i.), 0.17-0.75 [10]). On the other hand, in tumors that do eventually develop, the Pro-allele is predicted to be associated with an advanced, invasive, and metastatic disease, due to the ability of TGF $\beta$ to promote tumor progression at later stages by modulating the tumor microenvironment, enhancing invasiveness, and inhibition of immune cell function [1-4]. Indeed, patients who carried the Pro-allele had a significantly reduced 5-year disease-free survival rate compared to Leu/Leu patients [9]. In association studies, this effect should be reflected by odds ratios above unity in subpopulations with advanced stages, and in entire studies of patients with a predominantly advanced cancer stage. 
How is the "advanced stage", in which tumors are responsive to the tumor promoting activity of TGF $\beta$ but no longer to its initial tumor suppressive activity, reflected by clinical tumor parameters? TGF $\beta$ primarily enhances a tumor's invasive and metastatic potential, which are the major determinants of disease outcome [1-4]. Accordingly, we propose that markers of poor prognosis define an advanced stage in the context of TGF $\beta$ signaling. We indeed observed higher odds ratios associated with the Pro-allele in patients with pT2-4 vs. pT1 tumors, grade 2 vs. grade 1 tumors, and negative vs. positive ER status, although these trends were not significant at the $95 \%$ confidence level (data not shown). The BCAC study has reported higher odds ratios associated with the Pro-allele in patients with high tumor grade and stage, and negative ER and PR status, although only the latter association was statistically significant [6]. Similarly, the Pro-allele was associated with a reduced risk of early-stage breast cancer, but an increased risk of breast cancer with advanced stages [18]. We observed a significantly younger age at diagnosis of Pro/Pro patients compared to Leu/Leu patients, which could also be due to a faster breast cancer progression associated with the Pro-allele (Figure 1). This novel finding is consistent with a trend towards higher odds ratios associated with the Pro-allele in younger patients in the BCAC study [6].

\section{Experimental Section}

The study population has been described in detail in [27]. Briefly, 276 consecutive female breast cancer patients and 255 controls (patients with benign gynecological lesions and healthy female donors without breast cancer or any other malignancies) were enrolled between 2002 and 2004 at the Department of Obstetrics and Gynecology, Medical University of Vienna (MUV), Austria. Only women of Caucasian background from the same geographical area were included in this study. This study was approved by the institutional review board of the MUV, and written informed consent was obtained from all participants. For technical reasons, the genotype could not be determined for 2 patients and 3 controls. Thus, all further analyses were based on the remaining 526 subjects; their clinical and histopathological characteristics are shown in Table 1.

Genomic DNA was extracted from patients' blood with the QIAamp DNA Blood Midi kit (Qiagen, Venlo, the Netherlands) per the manufacturer's instructions. Genotyping of SNP rs1800470 (TGF $\beta 1$ L10P; Leu10Pro; T + 29C; formerly termed rs1982073) was performed by TaqMan PCR with allele-specific, fluorescently labeled probes following the manufacturer's instructions (Applied Biosystems, Brunn/Gebirge, Austria; Assay-ID \# C_22272997_10). 40 ng of genomic DNA were used per reaction in a total reaction volume of $10 \mu \mathrm{L}$.

Serum samples were collected from a subset of the patients and controls of this study between 2003 and 2004 under identical fasting conditions. None of the patients had undergone cancer treatment prior to serum isolation. Following genotyping, all available samples of patients $(n=25)$ and controls $(n=26)$ with the Pro/Pro genotype, and roughly equal numbers of randomly selected samples of the Leu/Leu and Leu/Pro genotypes were subjected to ELISA measurements of TGF $\beta 1$ concentrations with the Emax ImmunoAssay System (Promega, Madison, WI, USA). Prior to measurement, diluted serum samples were treated with hydrochloric acid, following the manufacturer's instructions to release any latent TGF $\beta 1$ from complexes with other serum proteins. 
Statistical analyses were performed with $\mathrm{R}$ version 2.15.1, an open-source language and environment for statistical computing [28]. Potential deviations of the study population from Hardy-Weinberg equilibrium were assessed with Chi-square tests with Yates' continuity correction. Differences between patients and controls, or between different genotypes, with respect to age or TGF $\beta 1$ serum concentrations were analyzed with unpaired, two-sided $t$-tests. Confidence intervals given are 95\% mid-P exact confidence intervals, i.e., considering all possible configurations of the contingency table that are more extreme than the observed configuration, and half the configurations that are equivalent to the observed one. Likewise, $p$-values shown in Table 2 are mid-P two-tailed exact $p$-values. Associations between the three L10P genotypes and clinical or histopathological characteristics were evaluated with Chi-square tests.

\section{Conclusions}

The current evidence is consistent with a model in which the Pro-allele of TGF $\beta 1$ is hypermorphic and reduces the overall incidence of breast cancer. However, if a tumor does develop in Pro-allele carriers, it is likely to progress faster to a more advanced stage, with invasive properties and a poor prognosis. Which of these conflicting effects predominates in a particular study likely depends on the specific composition of the study population. Our study had insufficient cases to detect a significant differential risk in subgroup analyses, thus it would be important to perform such analyses in larger studies and/or meta-analyses to better define the clinical subgroups in which the tumor-suppressive $v s$. tumor-promoting activities of TGF $\beta 1$ might be present.

\section{Acknowledgments}

This work was supported by funds of the "Oesterreichische Nationalbank" (Anniversary Fund, project number: 12994) and by the "Fellinger Krebsforschungsverein".

\section{Conflict of Interest}

The authors declare no conflict of interest.

\section{References}

1. Massague, J. TGFbeta in Cancer. Cell 2008, 134, 215-230.

2. Pardali, K.; Moustakas, A. Actions of TGF-beta as tumor suppressor and pro-metastatic factor in human cancer. Biochim. Biophys. Acta 2007, 1775, 21-62.

3. Siegel, P.M.; Massague, J. Cytostatic and apoptotic actions of TGF-beta in homeostasis and cancer. Nat. Rev. Cancer 2003, 3, 807-821.

4. Padua, D.; Massague, J. Roles of TGF-beta in metastasis. Cell Res. 2009, 19, 89-102.

5. Imamura, T.; Hikita, A.; Inoue, Y. The roles of TGF-beta signaling in carcinogenesis and breast cancer metastasis. Breast Cancer 2012, 19, 118-124.

6. Cox, A.; Dunning, A.M.; Garcia-Closas, M.; Balasubramanian, S.; Reed, M.W.; Pooley, K.A.; Scollen, S.; Baynes, C.; Ponder, B.A.; Chanock, S.; et al. A common coding variant in CASP8 is associated with breast cancer risk. Nat. Genet. 2007, 39, 352-358. 
7. Dunning, A.M.; Ellis, P.D.; McBride, S.; Kirschenlohr, H.L.; Healey, C.S.; Kemp, P.R.; Luben, R.N.; Chang-Claude, J.; Mannermaa, A.; Kataja, V.; et al. A transforming growth factor beta1 signal peptide variant increases secretion in vitro and is associated with increased incidence of invasive breast cancer. Cancer Res. 2003, 63, 2610-2615.

8. Lee, K.M.; Park, S.K.; Hamajima, N.; Tajima, K.; Yoo, K.Y.; Shin, A.; Noh, D.Y.; Ahn, S.H.; Hirvonen, A.; Kang, D. Genetic polymorphisms of TGF-beta 1 \& TNF-beta and breast cancer risk. Breast Cancer Res. Treat. 2005, 90, 149-155.

9. Shu, X.O.; Gao, Y.T.; Cai, Q.; Pierce, L.; Cai, H.; Ruan, Z.X.; Yang, G.; Jin, F.; Zheng, W. Genetic polymorphisms in the TGF-beta 1 gene and breast cancer survival: A report from the Shanghai Breast Cancer Study. Cancer Res. 2004, 64, 836-839.

10. Ziv, E.; Cauley, J.; Morin, P.A.; Saiz, R.; Browner, W.S. Association between the T29 $\rightarrow$ C polymorphism in the transforming growth factor $\beta 1$ gene and breast cancer among elderly white women: The study of osteoporotic fractures. JAMA 2001, 285, 2859-2863.

11. Cox, D.G.; Penney, K.; Guo, Q.; Hankinson, S.E.; Hunter, D.J. TGFB1 and TGFBR1 polymorphisms and breast cancer risk in the Nurses' Health Study. BMC Cancer 2007, 7, 175.

12. Feigelson, H.S.; Patel, A.V.; Diver, W.R.; Stevens, V.L.; Thun, M.J.; Calle, E.E. Transforming growth factor beta receptor type I and transforming growth factor betal polymorphisms are not associated with postmenopausal breast cancer. Cancer Epidemiol. Biomark. Prev. 2006, 15, 1236-1237.

13. Jin, Q.; Hemminki, K.; Grzybowska, E.; Klaes, R.; Soderberg, M.; Zientek, H.; Rogozinska-Szczepka, J.; Utracka-Hutka, B.; Pamula, J.; Pekala, W.; et al. Polymorphisms and haplotype structures in genes for transforming growth factor beta 1 and its receptors in familial and unselected breast cancers. Int. J. Cancer 2004, 112, 94-99.

14. Kaklamani, V.G.; Baddi, L.; Liu, J.; Rosman, D.; Phukan, S.; Bradley, C.; Hegarty, C.; McDaniel, B.; Rademaker, A.; Oddoux, C.; et al. Combined genetic assessment of transforming growth factor-beta signaling pathway variants may predict breast cancer risk. Cancer Res. 2005, 65, 3454-3461.

15. Krippl, P.; Langsenlehner, U.; Renner, W.; Yazdani-Biuki, B.; Wolf, G.; Wascher, T.C.; Paulweber, B.; Bahadori, B.; Samonigg, H. The L10P polymorphism of the transforming growth factor-beta 1 gene is not associated with breast cancer risk. Cancer Lett. 2003, 201, 181-184.

16. Le Marchand, L.; Haiman, C.A.; van den Berg, D.; Wilkens, L.R.; Kolonel, L.N.; Henderson, B.E. T29C polymorphism in the transforming growth factor betal gene and postmenopausal breast cancer risk: The Multiethnic Cohort Study. Cancer Epidemiol. Biomark. Prev. 2004, 13, 412-415.

17. Mu, L.; Katsaros, D.; Lu, L.; Preti, M.; Durando, A.; Arisio, R.; Yu, H. TGF- $\beta 1$ genotype and phenotype in breast cancer and their associations with IGFs and patient survival. Br. J. Cancer 2008, 99, 1357-1363.

18. Rebbeck, T.R.; Antoniou, A.C.; Llopis, T.C.; Nevanlinna, H.; Aittomaki, K.; Simard, J.; Spurdle, A.B.; Couch, F.J.; Pereira, L.H.; Greene, M.H.; et al. No association of TGFB1 L10P genotypes and breast cancer risk in $B R C A 1$ and $B R C A 2$ mutation carriers: A multi-center cohort study. Breast Cancer Res. Treat. 2009, 115, 185-192. 
19. Shin, A.; Shu, X.O.; Cai, Q.; Gao, Y.T.; Zheng, W. Genetic polymorphisms of the transforming growth factor-betal gene and breast cancer risk: A possible dual role at different cancer stages. Cancer Epidemiol. Biomark. Prev. 2005, 14, 1567-1570.

20. Sigurdson, A.J.; Hauptmann, M.; Chatterjee, N.; Alexander, B.H.; Doody, M.M.; Rutter, J.L.; Struewing, J.P. Kin-cohort estimates for familial breast cancer risk in relation to variants in DNA base excision repair, BRCA1 interacting and growth factor genes. BMC Cancer 2004, 4, 9.

21. Hishida, A.; Iwata, H.; Hamajima, N.; Matsuo, K.; Mizutani, M.; Iwase, T.; Miura, S.; Emi, N.; Hirose, K.; Tajima, K. Transforming growth factor B1 T29C polymorphism and breast cancer risk in Japanese women. Breast Cancer 2003, 10, 63-69.

22. Zheng, W. Genetic polymorphisms in the transforming growth factor-beta signaling pathways and breast cancer risk and survival. Methods Mol. Biol. 2009, 472, 265-277.

23. Guo, J.; Meng, H.; Pei, J.; Zhu, M. Association between the TNF-alpha-238G > A and TGF-beta1 L10P polymorphisms and breast cancer risk: A meta-analysis. Breast Care 2011, 6, 126-129.

24. Huang, Y.; Li, B.; Qian, J.; Xie, J.; Yu, L. TGF-beta1 29T/C polymorphism and breast cancer risk: A meta-analysis involving 25,996 subjects. Breast Cancer Res. Treat. 2010, 123, 863-868.

25. Yokota, M.; Ichihara, S.; Lin, T.L.; Nakashima, N.; Yamada, Y. Association of a T29 $\rightarrow$ C polymorphism of the transforming growth factor-betal gene with genetic susceptibility to myocardial infarction in Japanese. Circulation 2000, 101, 2783-2787.

26. Li, X.; Yue, Z.C.; Zhang, Y.Y.; Bai, J.; Meng, X.N.; Geng, J.S.; Fu, S.B. Elevated serum level and gene polymorphisms of TGF-beta1 in gastric cancer. J. Clin. Lab Anal. 2008, 22, 164-171.

27. Proestling, K.; Hebar, A.; Pruckner, N.; Marton, E.; Vinatzer, U.; Schreiber, M. The pro allele of the p53 codon 72 polymorphism is associated with decreased intratumoral expression of BAX and p21, and increased breast cancer risk. PLoS One 2012, 7, e47325.

28. R Development Core Team. R: A Language and Environment for Statistical Computing. $\mathrm{R}$ Foundation for Statistical Computing: Vienna, Austria, 2009.

(C) 2013 by the authors; licensee MDPI, Basel, Switzerland. This article is an open access article distributed under the terms and conditions of the Creative Commons Attribution license (http://creativecommons.org/licenses/by/3.0/). 